\title{
Space Debris: Flux in a Two Dimensional Orbit
}

\author{
David R. Brillinger*
}

\begin{abstract}
The debris circling the Earth has become an important environmental problem. In particular it creates nonnegligeable risk for spacecraft and satellites. This paper discusses a method to bound the rate of passage of objects through an arc segment in the plane of motion, given random initial conditions for the orbiting object. An advantage of the approach of this paper is that the initial conditions may be arbitrary and that moments beyond the first may be computed in a similar manner.
\end{abstract}

\section{Introduction}

There is now a substantial amount of debris in orbit about the Earth. It poses a risk to satellites and space craft and other debris. Unintended breakups as well as collisions occur. The objects in orbit include meteorites, solar system probes, functional spacecraft and debris made up of nonfunctional space craft, fragmentation debris and mission-related debris rocket bodies, $[6,8,9]$. Debilitating collisions have occurred, eg. an Ariane piece collided with a Cérise spacecraft, see [8]. Figure 1 is a NASA graphic illustrating some of the objects in low earth orbit at one point in time.

It is important to understand the risk associated with such debris. This paper is concerned with developing bounds for the intensity of passage of an object orbiting in a plane, through a short curve segment (or arc) in that

*Statistics Department, University of California, Berkeley, California, 94720-3860, USA 


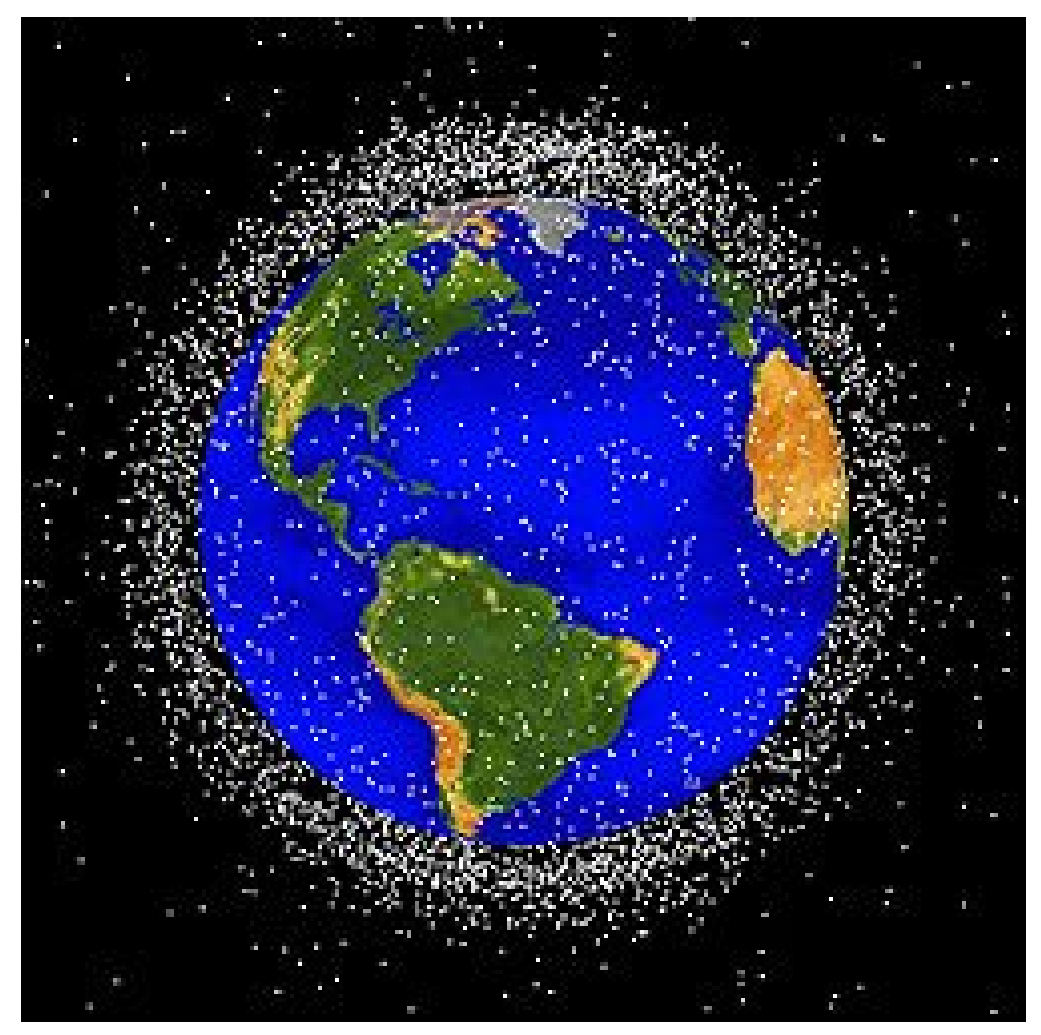

Figure 1: Debris in low earth orbit. www address: sn-callisto.jsc.nasa.gov

plane. The curve segments may be put together to form the boundary of an arbitrary shape and expressions obtained for risks of general objects.

This present paper refers to work in progress and in keeping with that situation is heuristic in approach.

\section{Space hazard}

Space debris risk assessment may be defined as: the estimation of the probability that certain performance variates, of a space object of interest, exceed relevant critical levels, within a specified time period, as a result of collisions with space debris. For example it may be of interest to estimate

$$
\text { Prob }\{\text { debris passes through }(\mathrm{x}, \mathrm{x}+d \mathbf{x}] \text { in time interval }[0, T]\}
$$


where $(\mathrm{x}, \mathrm{x}+d \mathbf{x}]$ refers to a small cell. It is clear that the time period of exposure is crucial to the description and estimation of such risks. The sizes of the debris pieces and of the object are also important in practice. In the present work $\mathrm{x}$ will be taken to lie in the orbital plane of the object.

\section{Risk probabilities}

A statistical concept that proves exceedingly useful in addressing problems of space risk is that of a point process, [4]. Realizations of such a process have the form $\left\{\mathrm{x}_{j}: j=1,2, \ldots\right\}$ with the $\mathrm{x}_{j}$ distinct points in a Euclidian space. In risk analyses the $\mathrm{x}_{j}$ refer to the locations of occurrence of some damaging event. Such a point process may also be described by a counting measure, with $N(A)=$ the number of points in a given set $A$. It is often convenient to work in terms of differentials $d N(A)=N(d A)$ where, for small $|d A|, d N(A)$ is 1 or 0 depending on whether or not there is a damaging event in the cell $d A$.

In risk analysis the probability of an event occuring in a prespecified set, $A$, is of particular importance. Under regularity conditions one can write

$$
\operatorname{Prob}\{N(A)>0\}=1-\sum_{k} \frac{(-1)^{k}}{k !} \int_{A} \ldots \int_{A} p_{k}\left(\mathbf{x}_{1}, \ldots, \mathbf{x}_{k}\right) d \mathbf{x}_{1} \ldots d \mathbf{x}_{k}
$$

where the $p_{k}\left(\mathbf{x}_{1}, \ldots, \mathbf{x}_{k}\right)$ are the $k$-th order product densities of the process, [4]. Product densities are defined as

$$
p_{k}\left(\mathrm{x}_{1}, \ldots, \mathbf{x}_{k}\right) d \mathbf{x}_{1} \ldots d \mathbf{x}_{k}=E\left\{d N\left(\mathbf{x}_{1}\right) \ldots d N\left(\mathbf{x}_{k}\right)\right\} \text { for the } \mathbf{x}_{j} \text { distinct }
$$

and $j=1, \ldots, k$. The expression (1) may be used to develop general bounds for risk, for example

$\int_{A} p_{1}(\mathbf{x}) d \mathbf{x} \geq \operatorname{Prob}\{N(A)>0\} \geq \int_{A} p_{1}(\mathrm{x}) d \mathbf{x}-\frac{1}{2} \int_{A} \int_{A} p_{2}\left(\mathbf{x}_{1}, \mathbf{x}_{2}\right) d \mathbf{x}_{1} d \mathbf{x}_{2}$

so the risk problem becomes one of approximating product densities, [5].

In the case of a Poisson process, with intensity function $p_{1}(\mathbf{x})=p(\mathbf{x})$, one has the exact expression

$$
\operatorname{Prob}\{N(A)>0\}=1-\exp \left\{-\int_{A} p(\mathbf{x}) d \mathbf{x}\right\}
$$


The Poisson is often pertinent to work with in risk problems because it arises as a limit when many independent point processes are superposed or when the points are rare, [4].

\section{Specifying orbits.}

Along the way to obtaining expressions for collision probabilities one needs a method to specify orbits. As Kepler and Newton showed these orbits are ellipses with the Earth at a focus, see Figure 2. In this paper the work will be for orbits within a given plane.

There are a number of coordinate systems. Refering to Figure 2 the following (partly classical) notation will be used:

$\mathrm{F}:$ a focus

FA: the base line

$\mathrm{O}$ : the center of the ellipse

$\mathrm{B}$ : the perigee, i.e. point on the orbit closest to $\mathrm{F}$

$a$ : the length of semimajor axis OB. $a>0$

$e$ : the eccentricity of the ellipse. $0 \leq e<1$

$\omega$ : the argument of perigee, i.e. the angle AFB. $0 \leq \omega<2 \pi$

$f$ : the true anomaly, i.e. the angle BFC. $0 \leq f<2 \pi$

$T$ : time of a perigee passage

$\mathrm{C}$ : the location of the object on its orbit at a given time, $t$

The elements, $a, e$, give the size and shape of the ellipse. The angles $\omega$ and $f$ are measured in the direction of motion of the object around the orbit. To fully specify the movement that direction also needs to be specified. The angle $\omega$ gives the orientation of the major axis relative to the base line FA.

Let $P$ denote the object's period. Suppose that $0 \leq T<P$, i.e. $T$ is the time of first perigee passage since time 0 . In this work it will be assumed that the initial condition for $(\omega, T)$ are stochastic eg. $\omega$ is uniform on $[0,2 \pi)$ and independently $T$ is uniform on $[0, P)$. Figure 3 provides a graph of some realizations of orbits for this process. The eccentricity is .9 .

The following coordinate system is particularly convenient for describing a particular location in the plane of motion: 


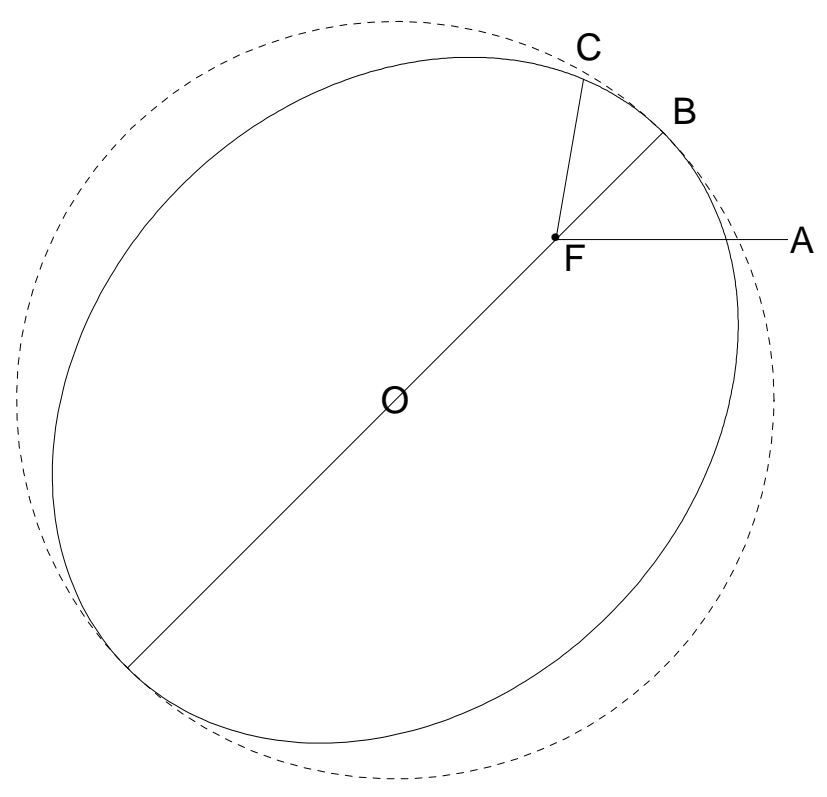

Figure 2: An orbit with eccentricity .6. The circumscribing circle is given as the curve of dashes. 


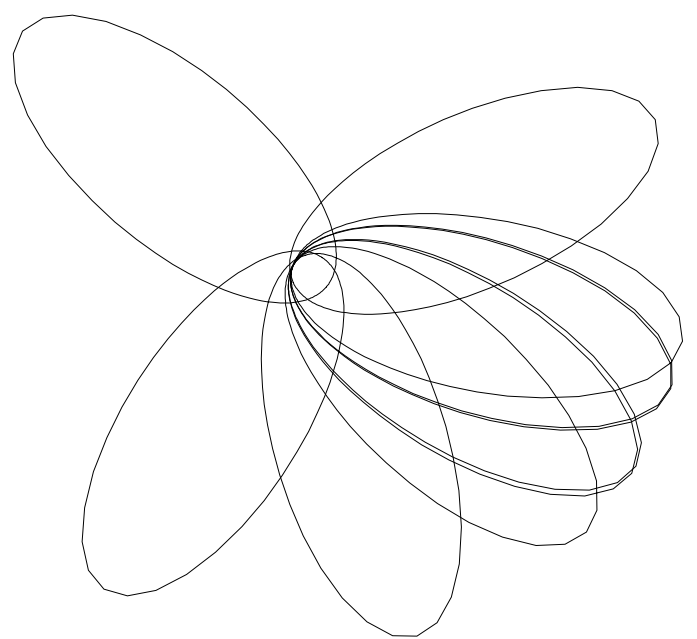

Figure 3: Some realizations of orbits for random initial conditions. 
$r$ : radial distance from the focus $F$ to the location $\mathrm{C}$ on the ellipse. $r>0$ $\phi$ : the angle AFC . $0 \leq \phi<2 \pi$

The value of $r$ is limited, $q<r<q^{\prime}$ where $q=a(1-e)$, and $q^{\prime}=a(1+e)$. At

time $t$ the object is at position $\mathrm{C},\left(\phi_{t}, r_{t}\right)$, with the angle $\mathrm{AFB}, \phi_{t}=\omega+f_{t}$.

The equations of motion are classic, [7, 11]. Suppose one is concerned with the location of the object on its orbit at time $t$. Let $E_{t}, 0 \leq E_{t}<2 \pi$ be defined via

$\cos f_{t}=\left(\cos E_{t}-e\right) /\left(1-e \cos E_{t}\right), \sin f_{t}=\sqrt{1-e^{2}} \sin E_{t} /\left(1-e \cos E_{t}\right)$

This $E_{t}$, is called the eccentric anomaly and is the angle BOD from the axis OB perpendicularly up to the point $\mathrm{D}$ on the circumscribing circle, passing through $\mathrm{C}$. The circle is given in the figure.

As time $t$ passes and $E_{t}$ goes from 0 to $2 \pi$ the the ellipse is swept out via

$$
r_{t}=a\left(1-e \cos E_{t}\right)
$$

In particular one has Kepler's equation relating $E_{t}$ to time $t$

$$
n(t-T)=E_{t}-e \sin E_{t}
$$

with $n$ the so-called mean motion. This equation has a unique solution for $E_{t}$ given the lefthand side, p. 192 in [2]. Equation (5) shows that the period of the object is given by $P=2 \pi / n$.

\section{Rate computation}

This work starts with the case of a single object orbiting in the plane. Its orbit may be denoted $\left(\phi_{t}, r_{t}\right), t \geq T$ where $\phi_{t}$ is the angle AFC and $r_{t}$ is the distance $\mathrm{FC}$. A probability of interest concerns the temporal rate of passage through a small arc, eg. one given parametrically by

$$
\{(\phi=a(u), r=b(u)), u \in U\}
$$

where say $U=[0,1]$ or $=(u, u+\Delta u)$. The arc might also be given as a graph, as in $u=\phi, r=b(\phi)$ or as in $\phi=a(r)$ for some range of $\phi$ or $r$ 
values. In a practical case, a satellite or space station might be described by a simply-connected curve.

The locations and times of passages through (6) lead to a two dimensional point process, $\left(t_{j}, u_{j}\right), j=1,2, \ldots$ with $a\left(u_{j}\right)=\phi_{t_{j}}, b\left(u_{j}\right)=r_{j}$. The point $\left(\phi_{t_{j}}, r_{t_{j}}\right)$ is the location of the $\mathrm{j}$-th passage through the arc and $t_{j}$ the time of that passage. If $N(t, u)=\#\left\{\left(t_{j}, u_{j}\right) \leq(t, u)\right\}$ denotes the point process, then the rate function

$$
\mu(t, u)=E\{d N(t, u)\} / d t d u
$$

is of interest. Given $\mu$ one can for example compute the expected flux through the arc (6) at time $t$ as

$$
\int_{U} \mu(t, u) d u / \int_{U} \sqrt{b^{\prime}(u)^{2}+b(u)^{2} a^{\prime}(u)^{2}} d u
$$

the denominator being the length of the arc.

The passages through the arc correspond to the solutions $(t, u)$ of the function pair $a(u)=\phi_{t}, b(u)=r_{t}$. There exists a convenient symbolic representation for the number of such solutions. First consider a real-valued differentiable function, $h(x)$, of a real variable, $x$, in an interval $U$. Its number of zeros there may be represented

$$
N(U)=\int_{U} \delta(h(x))\left|h^{\prime}(x)\right| d x
$$

where $\delta($.$) is the dirac delta function, [10]. For m$-vector-valued functions, $\mathbf{h}$, of an $m$-valued $\mathrm{x}$ and a region $A$ one has the analagous expression

$$
N(A)=\int_{A} \delta(\mathbf{h}(\mathbf{x}))|J \mathbf{h}(\mathbf{x})| d \mathbf{x}
$$

for the number of solutions of $\mathbf{h}(\mathbf{x})=\mathbf{0}$ in $\mathrm{A}$. This assumes $\mathbf{h}$ is Lipschitz and writes $J$ h for the Jacobian, [3].

To use (9) in the present context, take the 2 equations, $a(u)=\phi_{t}, b(u)=r_{t}$, in the 2 unknowns $t, u$, Supposing that the functions $a, b$ are differentiable, the Jacobian, $J \mathbf{h}$, is equal to

$$
a^{\prime}(u) \dot{r}_{t}-b^{\prime}(u) \dot{\phi}_{t}
$$

having written $\dot{\phi}, \dot{r}$ for $d \phi_{t} / d t, d r_{t} / d t$ and $a^{\prime}, b^{\prime}$ for $d a / d u, d b / d u$. 
Taking $A$ in (9) to be $(t, t+d t) \times U$ one has

$$
N(d t, U)=\left(\int_{U} \delta\left(\phi_{t}-a(u)\right) \delta\left(r_{t}-b(u)\right)\left|a^{\prime}(u) \dot{r}_{t}-b^{\prime}(u) \dot{\phi}_{t}\right| d u\right) d t
$$

Suppose that the variate $(\omega, T)$ has density function $p(\omega, T)$ with $0 \leq \omega<2 \pi$ and $0 \leq T<P$. Then the expected value of (10) is

$$
\begin{gathered}
\int_{E} \int_{U} p\left(a(u)-\operatorname{atan}\left(\sqrt{1-e^{2}} \sin E, \cos E-e\right), t-\frac{1}{n}(E-e \sin E)\right) \delta(a(1-\cos E)-b(u)) \\
\cdot\left|a^{\prime}(u) a \sin E-b^{\prime}(u) \frac{\sqrt{1-e^{2}}}{1-e \cos E}\right| d u d E d t
\end{gathered}
$$

with $q<b(u)<q^{\prime}$. Details of the derivations and some simplifications are given in the Appendix.

The following examples are useful supposing that $\omega, T$ are purely random, i.e. are respectively independent uniforms, $U(0,2 \pi), U(0, P)$. Their joint density $p(\omega, T)=1 /(2 \pi P)$.

Example 1. Suppose that the arc of interest is $u=\phi, a(u)=\phi, b(u)=r$ so $a^{\prime}(u)=1, b^{\prime}(u)=0$, i.e. one has an arc of the circle centered at $F$ of (constant) radius $r$. The angle $\phi$ is AFC. The distance $r$ is FC. An example is graphed in Figure 4. From expression (18) in the Appendix, taking $U$ to be $(\phi, \phi+\Delta \phi)$, the desired expected value is

$$
\frac{\Delta \phi}{\pi P}
$$

provided $q<r<q^{\prime}$. This does not depend on $t$ or $\phi$. If one is concerned with the whole circle the rate is $2 / P$, i.e. there are two crosses by the object per orbit.

Example 2. As a second example suppose that the segment of interest is $u=r, a(u)=\phi, b(u)=r$ so $a^{\prime}(u)=0, b^{\prime}(u)=1$. This is an interval on the radius originating at $F$ at angle $\phi$, see Figure 4. Taking $U$ to be $(r, r+\Delta r)$ the expected value, may be evaluated from expression (18) in the Appendix. It is found to be

$$
\frac{a \sqrt{1-e^{2}}}{r} \frac{1}{\sqrt{(r-q)\left(q^{\prime}-r\right)}} \frac{\Delta r}{\pi P}
$$



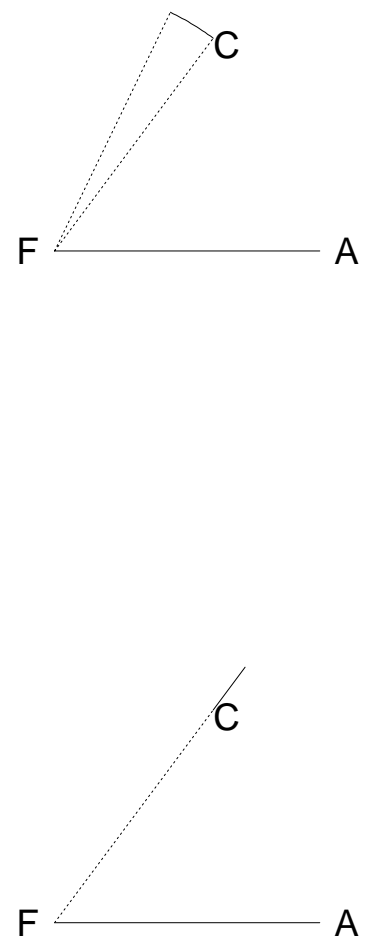

Figure 4: The arcs of Examples 1 and 2. 


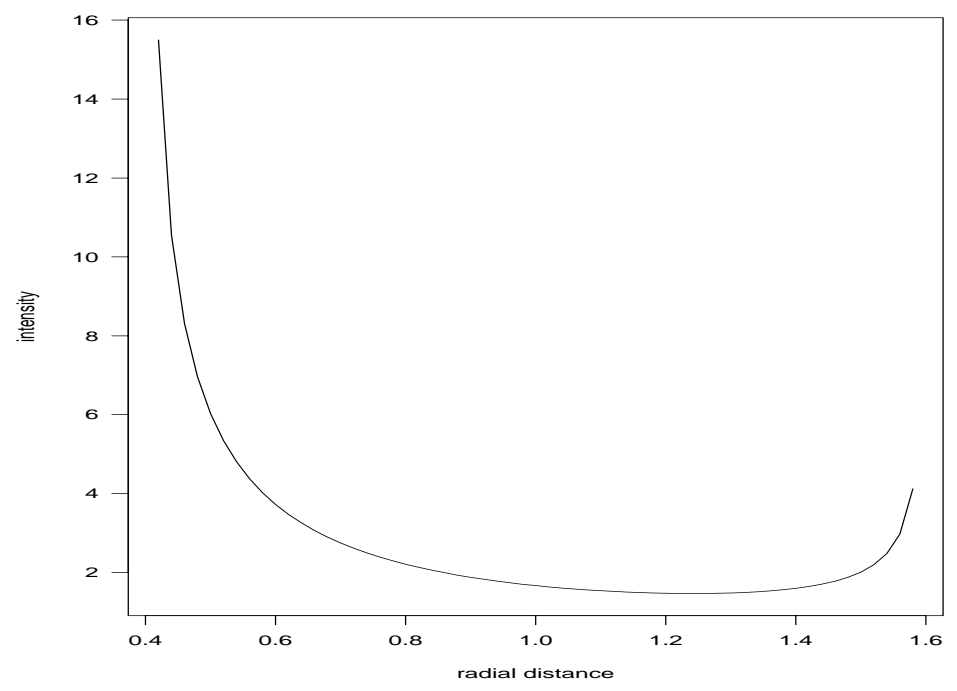

Figure 5: The rate function (13).

where $q, q^{\prime}=a(1-e), a(1+e), q<r<q^{\prime}, 0 \leq \phi<2 \pi$. This quantity does not depend on $t$ as could have been anticipated. It is graphed in Figure 5 for the case of eccentricity .6. The function depends strongly on $r$. One sees high intensity at the extremes, $q, q^{\prime}$, of the orbit with the highest at $q$.

If one inegrates this expression from $q$ to $q^{\prime}$ one obtains $1 / P$ consistent with the object crossing the line segment once per orbit.

\section{Discussion}

In practice there may be a number of objects orbiting simultaneously. Supposing their initial conditions are sampled independently from $p(\omega, T)$, the rate function remains proportional to that given by expression (11) and for a large number the overall process that is approximately Poisson, so one can obtain approximate risk probabilities as at (2).

Dependence may be introduced amongst particles by assuming a joint distribution for $\left(\omega_{1}, T_{1}\right)$ and $\left(\omega_{2}, T_{2}\right)$. This is necessary, for example, to handle particles resulting from a breakup. 
For a product density of order 2 one replaces expression (10) with

$$
N\left(d t_{1}, U\right) N\left(d t_{2}, U\right), t_{1} \neq t_{2}
$$

Its expected value may be evaluated in the manner of the one dimensional case. Examples will be provided in a later paper.

That the time origin is purely random, i.e. that $T$ is $U[0, P)$, seems plausible for many situations. But it does seem worth assuming that the distribution of $\omega$ is not uniform, for example a launch may be made with a prespecified $\omega_{0}$. Formulae (11) and (18) give results for $\omega$ having density $p(\omega)$.

Only the planar case has been considered in this paper. This is what is usually done to begin in the texts on orbital mechanics. The three dimensional case will be addressed in the next paper.

All the assumptions needed for the development of the results have not been presented, eg. conditions allowing exchange of orders of integration. One may avoid the use of th Dirac $\delta$ function by formal limiting arguments, as in $[3]$

\section{Acknowledgements}

This research received partial support from the NSF Grants DMS-9704739 and DMS-9971309. The author wishes to thank Abdel El Shaarawi for getting him involved with NASA's Haystack Review and the researchers at Johnson Space Center for their introduction to the problems involved.

\section{References}

[1] Barton, D.K., Brillinger, D. R., El-Shaarawi, A. H., McDaniel, P., Pollock, K. H. and Tuley, M. T. (1998). Final Report of the Haystack Orbital Debris Data Review Panel. Technical Memorandum 4809 NASA, Johnson Space Center, Houston.

[2] Battin, R. H. (1987). An Introduction to the Mathematics and Methods of Astrodynamics. American Institute of Aeronautics and Astronautics, 
New York.

[3] Brillinger, D. R. (1972). On the number of solutions of systems of random equations. Ann. Math. Statist. 43, 534-540.

[4] Brillinger, D. R. (1978). Comparative aspects of the study of ordinary point processes and of time series. pp. 33-133 in Developments in Statistics 1. Academic, New York.

[5] Brillinger, D. R. (1982). Some bounds for seismic risk. Bull. Bull. Seismol. Soc. America 72, 1403-1410.

[6] Cleghorn, G. et al. (1995). Orbital Debris: a Technical Assessment. National Academy Press, Washington.

[7] Geyling, F. T. and Westerman, H. R. (1971). Introduction to Orbital Mechanics. Addison-Wesley, Reading.

[8] Johnson, N. L. (1998). Monitoring and controlling debris in space. Scientific American, August, 62-67.

[9] Johnson, N. L. and McKnight, D. S. (1987). Artificial Space Debris. Orbit Book Co., Malabar, Florida.

[10] Kac, M. (1943). On the average number of real roots of a random algebraic equation. Bull. Amer. Math. Soc. 49, 314-320.

[11] Szebehely, V. G. (1989). Adventures in Celestial Mechanics. U. of Texas Press, Austin.

\section{Appendix}


In the next develpments it is convenient to work in terms of the angle $E_{t}$, indeed [11], p. 48 speaks of "the power of using the 'proper' variables in celestial mechanics".

Following expression (10) one wishes the expected value of

$$
\begin{gathered}
N(d t, U)= \\
\left(\int_{U} \delta\left(\phi_{t}-a(u)\right) \delta\left(r_{t}-b(u)\right)\left|a^{\prime}(u) \dot{r}_{t}-b^{\prime}(u) \dot{\phi}_{t}\right| d u\right) d t
\end{gathered}
$$

with the expected value taken over $\left(\phi_{t}, r_{t}, \dot{\phi}_{t}, \dot{r}_{t}\right)$. These variates need to be expressed in terms of $(\omega, T)$ since that is the source of the randomness.

One has equation (4)

$$
r_{t}=a\left(1-e \cos E_{t}\right)
$$

and from it

$$
\begin{gathered}
\dot{r}_{t}=\text { nae } \sin E_{t} /\left(1-e \cos E_{t}\right) \\
\text { since } \dot{E}_{t}=n /\left(1-e \cos E_{t}\right),[11] . \text { Next, as } \phi_{t}=\omega+f_{t}, \text { from }(3) \\
\phi_{t}=\omega+\operatorname{atan}\left(\sqrt{1-e^{2}} \sin E_{t}, \cos E_{t}-e\right)
\end{gathered}
$$

From this last

$$
\dot{\phi}_{t}=n \sqrt{1-e^{2}} /\left(1-e \cos E_{t}\right)^{2}
$$

In these terms (14) becomes

$$
\begin{aligned}
& \left(\int_{U} \delta\left(\omega+\operatorname{atan}\left(\sqrt{1-e^{2}} \sin E_{t}, \cos E_{t}-e\right)-a(u)\right) \delta\left(a\left(1-e \cos E_{t}\right)-b(u)\right)\right. \\
& \left.\cdot\left|a^{\prime}(u) n a e \sin E_{t} /\left(1-e \cos E_{t}\right)-b^{\prime}(u) n \sqrt{1-e^{2}} /\left(1-e \cos E_{t}\right)^{2}\right| d u\right) d t
\end{aligned}
$$

To continue, $E_{t}$ as a random variable, needs to be related to $(\omega, T)$. From (5) one has

$$
-n d T=\left(1-e \cos E_{t}\right) d E_{t}
$$

So the probability element

$$
p(\omega, T) d \omega d T
$$

$0 \leq \omega<2 \pi, 0 \leq T<P$ becomes

$$
p\left(\omega, t-\frac{1}{n}(E-e \sin E)\right) \frac{1}{n}(1-e \cos E) d \omega d E
$$


for $0 \leq E<2 \pi$.

For the moment it is convenient to write

$$
p_{t}(\omega, E)=p\left(\omega, t-\frac{1}{n}(E-e \sin E)\right)
$$

Using the above expressions, the expected value of (15) may be written

$$
\begin{gathered}
\left.\int_{E} \int_{U} \frac{1}{n}(1-e \cos E) p_{t}\left(a(u)-\operatorname{atan}\left(\sqrt{1-e^{2}} \sin E, \cos E-e\right), E\right)\right) \delta(a(1-\cos E)-b(u)) \\
\cdot\left|a^{\prime}(u) n a e \frac{\sin E}{1-e \cos E}-b^{\prime}(u) n \frac{\sqrt{1-e^{2}}}{(1-e \cos E)^{2}}\right| d u d E d t
\end{gathered}
$$

At this point it makes sense to break the integral over $E$ into the intervals $[0, \pi)$ and $[\pi, 2 \pi)$ and to use the transformation

$$
\cos E=\frac{1}{e}\left(1-\frac{v}{a}\right), \sin E= \pm \frac{1}{e a} \sqrt{(v-q)\left(q^{\prime}-v\right)}
$$

with the " + " $\operatorname{sign}$ for $0 \leq E<\pi$, and the "-" for $\pi \leq E<2 \pi$. In either case

$$
d E=\frac{1}{\sqrt{\left(q^{\prime}-v\right)(v-q)}} d v
$$

Now expression (?) is

$$
\begin{aligned}
& \int_{q}^{q^{\prime}} \int_{U} \frac{v}{a n} p_{t}\left(a(u)-\operatorname{atan}\left(\sqrt{1-e^{2}} \frac{1}{e a} \sqrt{(v-q)\left(q^{\prime}-v\right)}, \frac{1}{e}\left(1-\frac{v}{a}\right)\right), \operatorname{acos}\left(\frac{1}{e}\left(1-\frac{v}{a}\right)\right)\right. \\
& \cdot \delta(v-b(u)) \mid a^{\prime}(u) n a e \frac{1}{e a} \sqrt{(v-q)\left(q^{\prime}-v\right) \frac{a}{v}-b^{\prime}(u) \frac{n \sqrt{1-e^{2}}}{(v / a)^{2}}} \sqrt{\frac{1}{\left(q^{\prime}-v\right)(v-q)} d v d u d t} \\
& \int_{q}^{q^{\prime}} \int_{U} \frac{v}{a n} p_{t}\left(a(u)-a \tan \left(-\sqrt{1-e^{2}} \frac{1}{e a} \sqrt{\left.(v-q)\left(q^{\prime}-v\right), \frac{1}{e}\left(1-\frac{v}{a}\right)\right), a \cos \left(\frac{1}{e}\left(1-\frac{v}{a}\right)\right)}\right.\right. \\
& \cdot \delta(v-b(u)) \mid-a^{\prime}(u) n a e \frac{1}{e a} \sqrt{(v-q)\left(q^{\prime}-v\right)} \frac{a}{v}-b^{\prime}(u) \frac{n \sqrt{1-e^{2}}}{(v / a)^{2}} \sqrt{\frac{1}{\left(q^{\prime}-v\right)(v-q)}} d v d u d t
\end{aligned}
$$


In the case that $U=(u, u+\Delta u)$ and that $p(\omega, T)$, is constant, at $1 / 2 \pi P$, this becomes

$$
\begin{gathered}
\left(\mid a^{\prime}(u)-b^{\prime}(u) \frac{a}{b(u)} \frac{\sqrt{1-e^{2}}}{\sqrt{\left(q^{\prime}-b(u)\right)(b(u)-q)}}\right. \\
\left.+\left|-a^{\prime}(u)-b^{\prime}(u) \frac{a}{b(u)} \frac{\sqrt{1-e^{2}}}{\sqrt{\left(q^{\prime}-b(u)\right)(b(u)-q)}}\right|\right) \frac{\Delta u d t}{2 \pi P}
\end{gathered}
$$

provided $q<b(u)<q^{\prime}$. 